\title{
New EMC Effects with Multi-layered Type of EM Shield
}

\author{
Aron Tesfalem Berhe ${ }^{1}$ and Frank Gräbner ${ }^{2^{*}}$ \\ ${ }^{1}$ Karadeniz Technical University, Trabzon, 61080, Turkey \\ ${ }^{2}$ IMG Electronic and Power Systems GmBH, Nordhausen, 99734, Germany \\ *E-mail of corresponding author: frank.graebner@img-nordhausen.de
}

\begin{abstract}
Electro-magnetic interference is one of the biggest problems which hinder electrical and/or electronic devices from operating efficiently in addition to the negative impact it can have on environment. However, with proper shielding, unwanted electromagnetic interference can be substantially reduced, hence to achieve better EM compatibility among devices and avail safer environment. This paper presents multi-layered electromagnetic shield design analysis by considering different scenarios. The analysis is based on simulation done using MATLAB.
\end{abstract}

Received 14 September 2021

Accepted 28 October 2021

Published 22 December 2021

Keywords: electromagnetic interference, shielding effect, reflectivity, absorption.

\section{Introduction}

In today's time of EMC problems, the problems of new e-mobility applications are particularly present in the $\mathrm{kHz}$ range and lower $\mathrm{MHz}$ range.

The work presented is intended to specify a new EMC shielding concept that should improve the EMC shielding. This involves the use of multilayer shielding layers with absorbent intermediate layers.

Electromagnetic interference (EMI) originates from unwanted electrical or magnetic coupling between components in a module or system [1, 2, 3]. Fast switching circuits, communication devices, and electrical devices which operate with high frequency, are the main sources of unwanted electromagnetic radiations. This cause's electromagnetic interference (noise), which ends up in coupling with other components and degrading the performance of surrounding circuits which are sensitive to EM signals (Unwanted signals). Apart from this, if not properly contained, the impact could go further from affecting lives to causing unwanted destructions of entities.

Different shielding methods have been used so far and they proved to be effective. The types of materials which are usually scientifically proposed to be used in EM shielding are those with good magnetic (good permeability), electric (permittivity) and conductive (conductivity) behaviour. It comes into play because of their good response to electromagnetic waves.

Some of them could have better reflectivity behaviour, while some of them could have good EM absorption behaviour. Various types of shields including single layer, multi-layer conductors, and doped polymers with conductive behaviour are mentioned in literature with sole purpose of optimizing the performance of the shielding behaviour of materials. [2]

It has been proven that multilayer type of shield can

(C) The Author(s) 2021. Published by CIEES Association Press. This work is licensed under a Creative Commons Attribution-NonCommercialNoDerivs 4.0 Generic License. provide better shielding by manipulating the advantage of their distinct features to the overall contribution of the design.

Some of the challenges which are faced when designing a multilayer EM shield are determining the optimum thickness of the layers used, their spatial arrangement and their intrinsic chemical behaviour. Because a perfect or optimum combination of these factors results in very high shielding effect or very low EMI, hence better electromagnetic compatibility.

It is well known that, when designing metal packaging for EM shielding purposes, frequency resonance have negative impact the shielding performance because it reduces the shielding effectiveness. Hence, in this paper, different cases have been discovered to check if it is possible to reduce this problem and achieve optimum shielding effectiveness.

Ferrite absorber, reflector (Conductive polymer) and conductor (steel) are going to be considered. Near field application has been considered. Different scenarios have been considered, mainly focusing on the geometry and spatial arrangement of the layers.

\section{Principle}

Shielding effect is the sum of three parameters and can be represented as follows:

$$
S=A+R+B,
$$

where $S=$ Shielding Effect, $A=$ Absorption, $R=$ Reflection, $B=$ Correction factor (Multiple reflection) [2]

Usually, major role is played by the absorption loss and the reflection loss. Hence only transmission characteristics and reflectivity behaviour are considered. [4]

Since the aim of this experiment is to test multilayered type of shield, the following model can be used to describe the general geometric arrangement of the layers used to construct any multi-layered EM shield.

As shown on Figure 1, an EM shield can be constructed with multilayers with each layer consisting 
different type of material with distinct characteristics. This improves the shielding effectiveness.

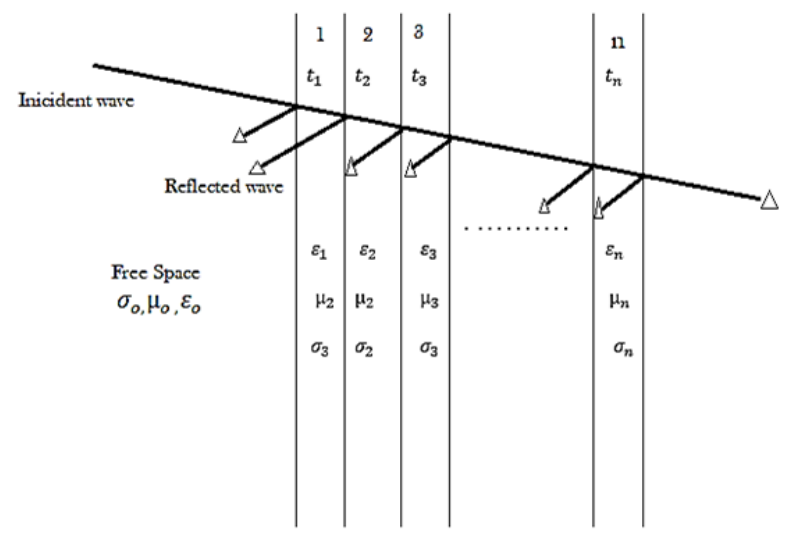

Figure 1. Multi-layer EM Shield Model [2].

The parameters $\varepsilon, \mu, \sigma$ represent permittivity, permeability and conductivity of each medium involved. It should be put in mind that the mediums used are assumed to be continuous and isotropic in order to make the analysis easier.

Shielding effect of a multi-layer shield is expressed as follows:

$S=-20 \log (T) d B$

where ' $T$ ' is the total transmission coefficient of $n$ interfaces for the n-layers which are overlaid on each other to form a laminate. Hence the following general equation could be used to calculate the transmission coefficient $[1,2,5]$ :

$$
\begin{aligned}
T & =P\left[\left(1-q e^{\left(-2 \gamma_{1} t_{1}\right)}\right)\left(1-q e^{\left(-2 \gamma_{2} t_{2}\right)}\right)\left(1-q e^{\left(-2 \gamma_{3} t_{3}\right)}\right)\right. \\
& \left.\ldots\left(1-q e^{\left(-2 \gamma_{n} t_{n}\right)}\right)\right] e^{\left(-\gamma_{1} t_{1}-\gamma_{2} t_{2}-\gamma_{3} t_{3} \ldots-\gamma_{n} t_{n}\right)}
\end{aligned}
$$

From above $t$ is the thickness of the layer and $P$ is as follows:

$$
P=\frac{\left(2 \eta_{0}\right)\left(2 \eta_{1}\right)\left(2 \eta_{2}\right)\left(2 \eta_{3}\right) \ldots\left(2 \eta_{n}\right)}{\left(\eta_{0}-\eta_{1}\right)\left(\eta_{1}-\eta_{2}\right)\left(\eta_{2}-\eta_{3}\right) \ldots\left(\eta_{n-1}-\eta_{n}\right)},
$$

Where $\eta_{0}$ is intrinsic impedance of free space which is equal to $120 \pi$ and $\eta_{1}, \eta_{2}, \eta_{3}$ and $\eta_{\mathrm{n}}$ are intrinsic impedances of layers $1,2,3$ and $n$ respectively. In this analysis, absorber, conductive polymer and conductor are considered. Their intrinsic impedances are calculated as follows: $[1,2,6]$

$$
\begin{aligned}
& \eta_{A}=\sqrt{\frac{-(2 \pi f \mu)^{2}}{\gamma A^{2}}} \text { (Intrinsic impedance of Absorber), } \\
& \eta_{p}=(1+j) \sqrt{\frac{\pi f \mu_{p}}{\sigma_{p}}} \text { (Intrinsic Impedance of conductive polymer), } \\
& \eta_{c}=(1+j) \sqrt{\frac{\pi f \mu_{c}}{\sigma_{c}}} \text { (Intrinsic Impedance of conductor), }
\end{aligned}
$$

Each layer has its own distinct propagation constant $\gamma$ is defined as below:

$$
\gamma=(1+j) \sqrt{\pi f \mu \sigma}
$$

where $f=$ frequency, $\mu=$ Permeability of the medium and $\sigma=$ Conductivity of the medium. Finally $q$ stands for reflection coefficient at the interface of the layers and is computed as:

$q=\frac{\left(\eta_{n}-\eta_{n-1}\right)\left(\eta_{n}-Z(n+1)\right)}{\left(\eta_{n}+\eta_{n-1}\right)\left(\eta_{n}+Z(n+1)\right)}$,

where $Z(n)$ represents the impedance to the right of the nth interface. It is given as:

$$
Z(n)=\eta_{n}\left[\frac{Z(n+1) \cosh \left(\gamma_{n} t_{n}\right)+\eta_{n} \sinh \left(\gamma_{n} t_{n}\right)}{\eta_{n} \cosh \left(\gamma_{n} t_{n}\right)+Z(n+1) \sinh \left(\gamma_{n} t_{n}\right)}\right]
$$

As it has been mentioned above three layers, absorber, and conductive polymer apart from air or free space $[7,8,9]$. The material type parameter of each layer permittivity, permeability and conductivity are given in Table1.

In this paper, three layered and four layered EM shields has been considered. The simulation is done under different scenarios in which they are listed as follows:

- Effect of conductor layer thickness on the shielding effect of the overall three layered EM shield design;

- Effect of spatial arrangement of the different layers on the shielding effect (mainly absorber and conductive polymer);

Table 1. The material type parameter of each layer permittivity, permeability and conductivity.

\begin{tabular}{lccc}
\hline \multicolumn{1}{c}{ Material } & Permittivity & Permeability & Conductor \\
\hline Air (Free space) & $\varepsilon_{0}=8.854 * 10^{-12} \mathrm{~F} / \mathrm{m}$ & $\mu_{0}=4 \pi * 10^{-7} \mathrm{H} / \mathrm{m}$ & $\sigma_{0}=0$ \\
$\begin{array}{l}\text { Absorber } \\
\text { (Ferrite) }\end{array}$ & $\varepsilon_{r}=1.4+1.8 j$ & $\mu_{r}=22+19 j$ & \\
Conductive & $\left(1.24^{-11}+1.59^{-11} j\right) \mathrm{j} / \mathrm{m}$ & $\mu_{a}=(2.76+2.388 j) \mathrm{H} / \mathrm{m}$ & $\sigma_{a}=10^{-5} 1 / \Omega m$ \\
polymer & $\varepsilon_{r}=1.4+1.8 j$ & $\mu_{r}=1400+1500 j$ & \\
$\begin{array}{l}\text { Conductor } \\
\text { (Carbon Steel) }\end{array}$ & $\varepsilon_{p}=\left(1.24^{-11}+1.59^{-11} j\right) \mathrm{F} / \mathrm{m}$ & $\mu_{p}=(0.0018+0.0019 j) \mathrm{H} / \mathrm{m}$ & $\sigma_{p}=10^{-15} 1 / \Omega m$ \\
\hline
\end{tabular}


- Effect of adding air gap between the conductor and other two layers (In this paper the air gap itself is treated as a layer, in other words it is assumed as a 4 layered EM shield design);

- Effect of air gap thickness at constant frequency values;

- Effect of spatial arrangement of the layers on the shielding effect (Mainly absorber and conductive polymer).

\section{Results and discussion}

As shown in Figure 2, three random conductor thickness values has been considered at $\mathrm{t}=0.001 \mathrm{~m}$, $\mathrm{t}=0.0015 \mathrm{~m}, \mathrm{t}=0.0017 \mathrm{~m}$. It is found that the shielding effect increases with increase in the thickness of the conductor. However, as the thickness of the conductor increases, the frequency range of operation of the shield decreases i.e. as shown in the diagram the red line (lowest conductor thickness value) has highest frequency range, while the blue and green lines (with higher conductor thickness values) have slightly lower frequency range.

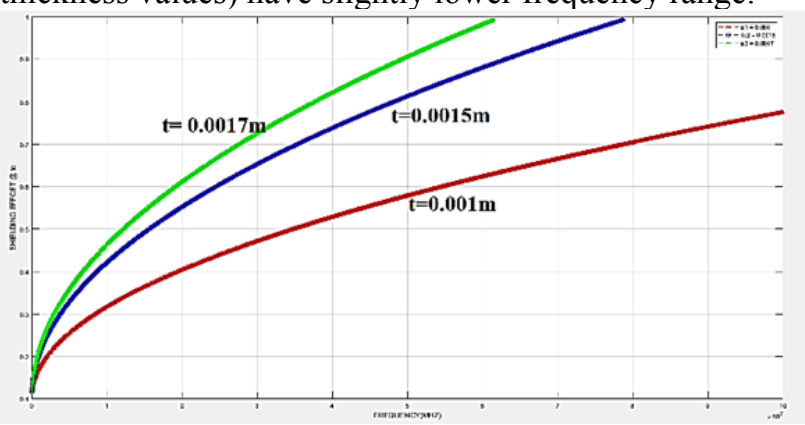

Figure 2. Shielding effect of three layered design at different thickness values.

In Figure 3, it shows the effect of spatial arrangement of the layers used in a three layered EM shield. The first arrangement is Absorber - Conductive Polymer Conductor (Blue) while the second one (Red) is Conductive polymer - Absorber - Conductor (Wave is assumed to propagate from left to right).

The effect is almost the same but when the result is magnified, it is found that the first case operates better (It is common to see such spatial arrangement used in literature especially for Multilayered EM shield design analysis).

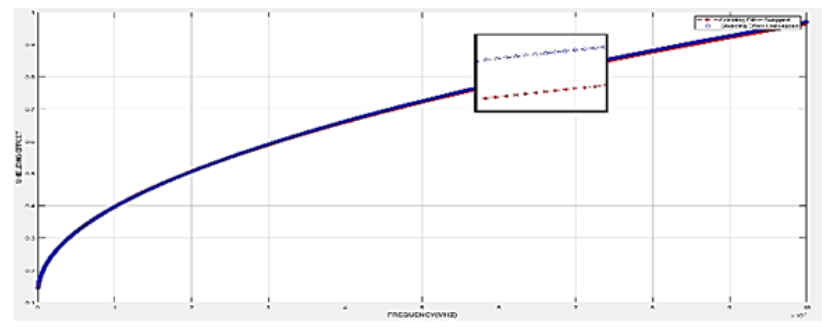

Figure 3. Effect of spatial arrangement shielding effect of three layered design.

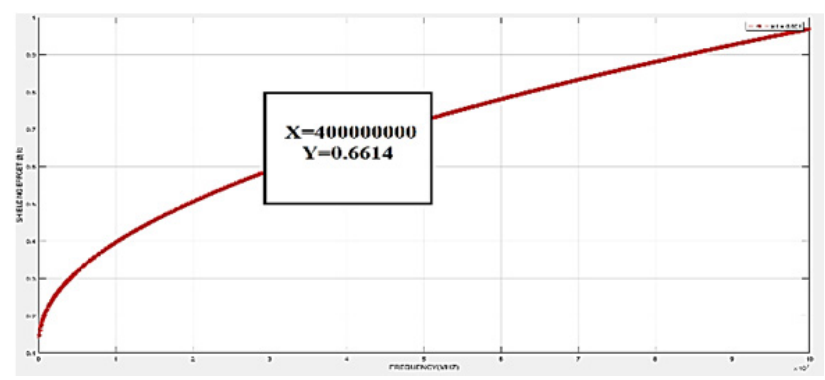

Figure 4. Shielding effectiveness of three layered EM shield design.

In the Figure 5, a three layer and four layer (Air-gap has been introduced inside) EM shields are considered.

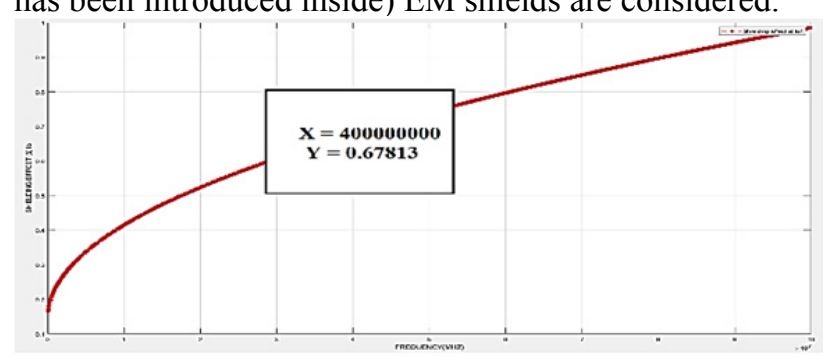

Figure 5. Shielding effectiveness of four layered EM shield design (Air-Gap is introduced between the conductor and the other two layers).

When compared with each other the performance of the EM shield with air-gap is better than without the airgap. To prove this the shielding effectiveness at $400 \mathrm{MHz}$ is chosen randomly and the plot shows that with air gap, shielding effect is about $0.6614 \mathrm{~dB}$ (normalised value to max shielding of $1 \mathrm{~dB}$ ) while without air-gap, it shows about $0.67813 \mathrm{~dB}$ (normalised value to max shielding of $1 \mathrm{~dB})$.

Figure 6 shows the effect of air gap thickness at constant frequency when air-gap is introduced. It is clearly shown that when the air-gap thickness varies, the shielding effect almost remains the same (the effect very small). On the other hand the effect of the frequency can be shown clearly, each line represents a constant frequency and this tells that, the EM shield performs better with increase in frequency keeping the air-gap thickness range constant

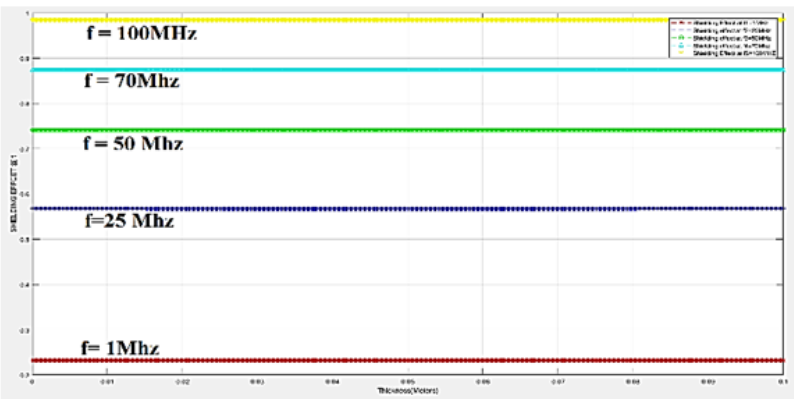

Figure 6. Shielding effectiveness vs. Thickness of air gap (keeping frequency constant) of four layered EM shield. 


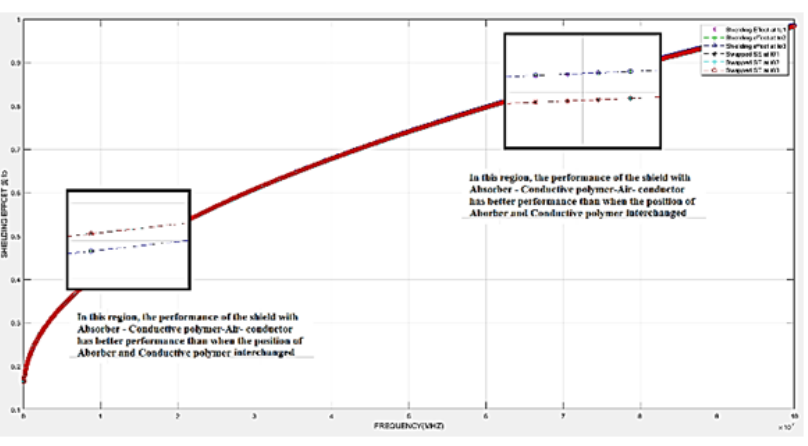

Figure 7. Effect of spatial arrangement shielding effect of four layered design (Blue: Absorber- Conductive Polymer-

Conductor, Red: Conductive Polymer- Absorber-Conductor).

In this case (Figure 7), the effect of geometric arrangement of the Absorber and Conductive- polymer is analysed. Although there is very small difference, the magnified plot shows that Conductive Polymer Absorber - Conductor (Red line) performs arrangement performs better in the lower part of the frequency range given while Absorber- Conductive Polymer- Conductor (Blue line) performs better in the higher part of the frequency range.

\section{Experimental Results}

The first indications of EMC shielding attenuation were measured with a monolayer.

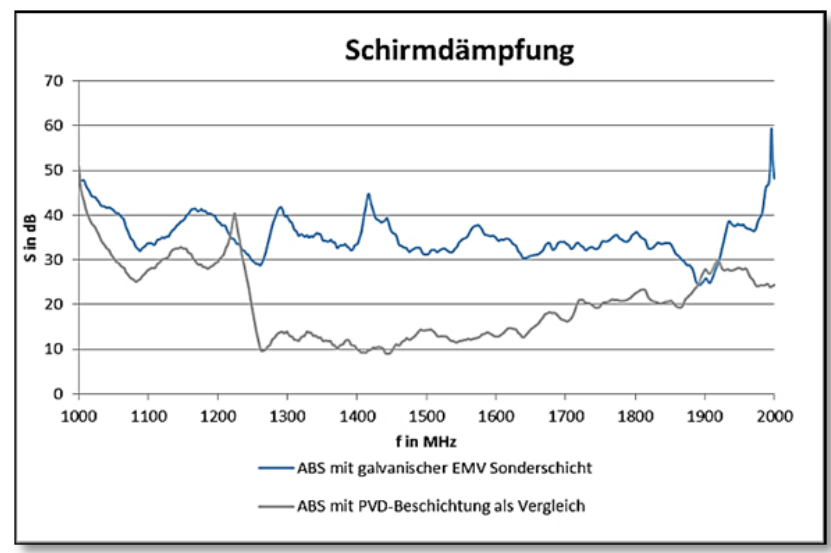

Figure 8. Shielding measurement result of a galvanic Monolayer [11].

In future EMC applications, multilayer layers will be built up and measured experimentally with regard to the shielding attenuation.

\section{Conclusion and future work}

Various cases have been discussed in this paper. From this it can be concluded that:

- Increase in conductor layer thickness improves the shielding effect. However, selecting the thickness needs carefully to be done because a specific frequency range can allow certain range of thickness for optimum shield effectiveness.

- Spatial arrangement of the layers in a multilayer EM shield has small effect on the shielding effectiveness, but it is advisable to design a shield Absorber -Conductive
polymer-Conductor mode (Assuming the incident EM wave touches absorber first). It is possible to find contradictory result (Figure 7), but this could be modified with a good design approach.

- Multilayer shield with air gap inside has better performance than without an air gap. But its performance response high to frequency than the thickness of the air gap.

In the future, it is possible to create a robust algorithm accrued with GUI, which can compute complex shielding effect calculations. This could simplify the rigorous calculation and simulation done in pre-design stage by accepting any intrinsic parameter values of the materials used in Electromagnetic shield design.

\section{References}

[1] Raj C. D., Rao G. S., Jayasree P. V. Y., Srinu B. \& Lakshman P. (2010). Estimation of reflectivity and shielding effectiveness of three layered laminate electromagnetic shield at X-Band, Progress In Electromagnetic Research B, vol. 20, pp. 205-223, doi:10.2528/PIERB10030402.

[2] Jayasree P., Surya Baba V., Prabhakar Rao B. \& Lakshman P. (2010). Analysis of Shielding Effectiveness of Single, Double and Laminated Shields for Oblique Incidence of EM Waves, Progress In Electromagnetics Research B, vol. 22, pp. 187-202, doi:10.2528/PIERB10051305.

[3] Kodali V. P. (2000). Engineering Electromagnetic Compatibility, Principles, Measurements and Technologies, S Chand and Company Ltd.

[4] Schulz R., Plantz V. \& Brush D. (1988) Shielding theory and practice, IEEE Transactions on Electromagnetic Compatibility, vol. 30 no.3, pp 187-201, doi: 10.1109/15.3297.

[5] Jayasree P., Baba V. \& Rao B. (2008). Shielding Effectiveness of Laminated Shields, Radioengineering, vol.17 no.4.

[6] Meshram M., Agrawal N., Sinha B. \& Misra P. (2003). Transmission line modeling (TLM) for evaluation of absorption in ferrite based multi layer microwave absorber TENCON 2003. Conference on Convergent Technologies for Asia-Pacific Region, Bangalore, India, pp 626-630 2, doi: 10.1109/ TENCON.2003.1273246.

[7] Feng Y., Qiu, T., Li X. et al. (2007). Microwave absorption properties of the carbonyl iron/EPDM radar absorbing materials, J. Wuhan Univ. Technol. vol. 22, pp 266270, doi.org/10.1007/s11595-005-2266-9.

[8] Praveen S., Babbar V., Razdan A., Srivastava S. \& Goel T. (2000). Microwave absorption studies of $\mathrm{Ca}-\mathrm{NiTi}$ hexaferrite composites in X-band, Materials Science and Engineering: B, vol.78 no.2-3, pp 70-74, doi: 10.1016/S0921-5107(00)00511-0. [9] Han-Shin C. \& Sung-Soo K. (1999). M-hexaferrites with planar magnetic anisotropy and their application to highfrequency microwave absorbers, IEEE Transactions on Magnetics, vol. 35 no. 5, pp 3151-3153, doi: $10.1109 / 20.801111$.

[10] Watanabe A., Raj P., Wong D. et al. (2018). Multilayered Electromagnetic Interference Shielding Structures for Suppressing Magnetic Field Coupling, Journal of Elec Materi vol. 47, pp. 5243-5250, doi: 10.1007/s11664-018-6387-2

[11] Gräbner F., Nath P. \& Hofinger J. (2019). WirelessAnwendungen in E-Antrieben störsicher betreiben, Galvanische EMV-Sonderschicht für 1000 bis $2000 \mathrm{MHz}$, available: https://www.all-electronics.de/wireless-anwendungen-in-eantrieben-stoersicher-betreiben. 